\title{
Design and Analysis of Equilateral Triangular Single Patch Antenna with Side Length $8 \mathrm{~cm}$
}

\author{
Ngu Sze Song, Kho Lee Chin, David Bong Boon Liang and Shamsiah bt Suhaili \\ Electronics Engineecring Department, Faculty of Engineering, Universiti Malaysia Sarawak (UNIMAS), 94300 Kota Samarahan, Sarawak. \\ ssngu@feng.unimas.my, lckho@feng.unimas.my, bbldavid@feng.unimas.my, sshamsiah@feng.unimas.my.
}

\begin{abstract}
Nowadays, different types of antenna were designed by using different parameters. Researchers take into consideration different parameters such as radiation pattern, power handling capability, frequency range, bandwidth, power gain, polarization, beam width, coverage, input impedance and the others when designing an antenna. The antenna's practicability, efficiency and cost remain the most critical issues in antenna design. In this paper, the details regarding antenna design parameters and considerations are shown. The design of equilateral triangular single patch antenna with length $8 \mathrm{~cm}$ and other suitable choices of thickness, material of patch and feeding technique is presented. Microwave Office software is used throughout the research for the simulation data modeling and analysis. The simulated resonant frequency is compared to the calculated resonant frequency.
\end{abstract}

Keywords: Patch Antenna; Feeding Technique; Resonant Frequency.

\section{Introduction}

Since the development and practical applications of log periodic and frequency independent antennas, a wide range of truly broadband antennas has been introduced to meet the varying demands imposed by specific requirements, such as area coverage and point to point circuits. Choosing and designing the right type of antenna design for use in a specific link is very critical to obtain the best link performance. To differentiate among the various antennas available, an understanding of the basic parameters of antennas performance and the ways this information can be presented is vital. The information of antenna parameters is also important for antenna design consideration in order to achieve the optimum outcome

\section{Microstrip Antenna}

Microstip antennas are also known as printed antennas. There are several types of microstrip antennas in which the most common on is the patch antenna. A patch antenna is a narrowband, wide-beam antenna fabricated by etching the antenna element pattern in metal trace bonded to an insulating substrate [1]. Patch antennas incorporate the ability to have polarization diversity. With a single feedpoint, patch antennas can easily be designed to have vertical, horizontal, right hand circular polarization (RHCP) or left hand circular polarization (LHCP). This unique property allows patch antennas to be used in many areas of communications link with varied requirements. Patch antennas have a very low profile, are mechanically rugged and can be conformable. Hence, they are often mounted on the exterior of aircraft and spacecraft and are incorporated into mobile radio communications devices.

Microstrip antennas are relatively inexpensive to manufacture and design due to the simple 2dimensional physical geometry. They are usually utilized at ultra-high frequency (UHF) and higher frequencies because the size of the antenna is directly tied to the wavelength at the resonant frequency. It had been generally applied in wireless communication system [2], [3].

The idea of using computer-based optimization for design tasks has been applied to many problems, including antenna design [4] with some predefined limitations.

\section{Analysis of Antenna's Performance}

Information that can be retrieved from antenna was crucial in determining the performance of antenna. In this paper, the performance of the designed antenna was analyzed according to few important data.

\subsection{Return Loss}

The return loss indicates how much of the incident power is reflected from the antenna. It is measure in decibel $(\mathrm{dB})$ unit. If $50 \%$ of the signal is absorbed by the antenna and $50 \%$ is reflected back, the return loss is $-3 \mathrm{~dB}$. A good antenna might have a value of $-10 \mathrm{~dB}$ ( $90 \%$ absorbed and 10\% reflected). The return loss of an antenna is given by:

$$
\text { Return Loss }(\mathrm{dB})=10 \log _{10} \operatorname{Pr}
$$

